\title{
Study on the Dynamic Prediction of Atomic Clock Frequency based on $\mathrm{UTCr}$
}

\author{
M. YAN \\ University of Chinese Academy of Sciences, Beijng, China; National Time Service Center, Chinese Academy \\ of Science , Xi'an, China; Key Laboratory of Time and Frequency Standards, Chinese Academy of Science, \\ $X i$ 'an, China. \\ L.L. QU\& S.W. Dong \& S.H. Zhao \\ National Time Service Center, Chinese Academy of Science, Xi'an, China; Key Laboratory of Time and \\ Frequency Standards, Chinese Academy of Science, Xi'an, China.
}

\begin{abstract}
The traditional atomic clock frequency prediction method has the characteristic of hysteresis which based on the clock frequency published by BIPM monthly. A dynamic prediction of atomic clock frequency model is proposed in this paper by using a rapid Coordinated Universal Time (UTCr) published by BIPM. The new method and the traditional method are applied respectively to the calculation of the atomic time scale in this paper. The time scale is calculated with the data of NICT, NTSC, NIM which participate in the calculation of International Atomic Time(TAI). The time scale calculated with the new method and the traditional method are compared with UTC-UTC $(k)$, respectively. The result shows that the new prediction method is more available than the traditional method. It improves greatly the quasi-real time of frequency prediction, provides more quasi-real time and reliable reference for frequency steering and contributes to getting better stability and accuracy of $\operatorname{UTC}(k)$.
\end{abstract}

KEYWORD: UTCr; frequency prediction; time scale.

\section{INTRODUCTION}

The Coordinated Universal Time (UTC) has been calculated with one-month data, and has been published monthly in the Circular T[1] under the form of UTC-UTC $(k)$ at five-day intervals from 1988. UTC is not adapted for real and quasi-real time applications. Therefore, a rapid Coordinated Universal Time UTCr is proposed by BIPM. The Time Department conducted an experiment of producing a "rapid UTC" ( UTCr) from January 2012 to the end of June 2013. About 40 laboratories contributed to the experiment. The BIPM Time Department produced an interim report in September 2012 and a final report in April 2013 for the Consultative Committee for Time and Frequency (CCTF)[2]. The report showed that UTCr had achieved the expected quality. Starting from July 2013, UTCr was published by BIPM on every Wednesday without interruption .The lagging is $3 \sim 10$ days which is greatly shorter than that of UTC (10 45 days).

The publication of UTCr will not change the calculation program of UTC monthly, UTC is still the only key of International time scale. UTCr has a positive impact on laboratories participating in calculation of TAI[3]: Laboratories which contributed to UTC would have more frequent assessing of the $\mathrm{UTC}(k)$ steering; Users of $\mathrm{UTC}(k)$ would have access to a better 'local' reference, and indirectly, better traceability to the UTC 'global' reference. UTCr is not a time scale which exists independently of UTC.Rather, UTCr can provide a reference for prediction of clock frequency and drift. Also, it can enhance the International cooperation of time laboratories.

For clocks of different laboratories, their frequency prediction bases on the clock frequency published by BIPM monthly with a certain hysteresis. At the same time, due to the influence of many kinds of noises[4], clock frequency unable to be quasi-real time forecasted. Thus, it is difficult to obtain better stability and accuracy of $\operatorname{UTC}(k)$ by frequency steering. UTCr is published officially by BIPM every week from July 2013 that reached the anticipated quality and the lagging of it is $3 \sim 10$ days. It provides reference for the accurate prediction of clock frequency.

\section{FREQUENCY PREDICTION MODEL}

The clock frequency of the last interval which is published by BIPM is considered as the prediction of the current interval in the traditional frequency prediction method. Calculate the time scale providing reference for $\mathrm{UTC}(k)$ steering. Due to the characteristics of atomic clock and the lagging 
(10 45 days) of Circular $\mathrm{T}$, it is infeasible to predict real or quasi-real time clock frequency, however, the publication of UTCr helps to improve the situation. At present, the time difference between $\mathrm{UTCr}-\mathrm{UTC}(k)$ and $\mathrm{UTC}-\mathrm{UTC}(k)$ of the International advanced laboratories is kept within $\pm 2 \mathrm{~ns}$ and the curves of $\mathrm{UTCr}-\mathrm{UTC}(k)$ and $\mathrm{UTC}-\mathrm{UTC}(k)$ have a consistent trend. As a conclusion, it is available to predict clock frequency based on UTCr and to calculate the time scale, consequently to obtain better stability and accuracy of $\operatorname{UTC}(k)$.

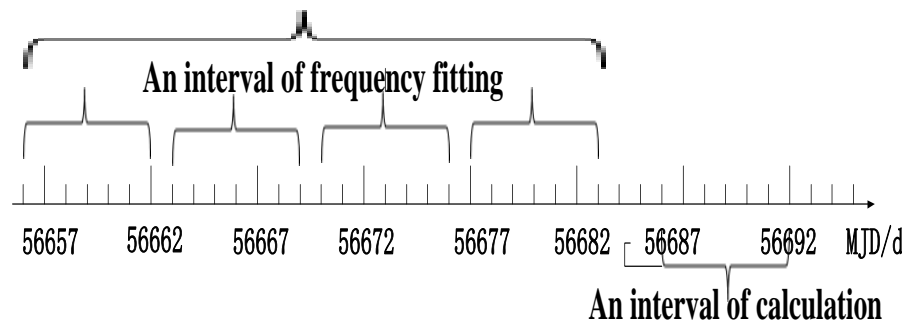

Figure 1.The diagram of an interval of frequency fitting and an interval of calculation.

As shown in the Fig .1, the horizontal axis represents Modified Julian Date, each parenthese represents $\mathrm{UTCr}-\mathrm{UTC}(k)$ published by BIPM on every Wednesday. Calculate the time difference of $\mathrm{UTCr}-\operatorname{Clock}(i)$ by $\mathrm{UTCr}-\mathrm{UTC}(k)$ of the four weeks before and the clock data UTC $(k)-\operatorname{Clock}(i)$ collected by equipments, that is:

$\mathrm{UTCr}-\operatorname{Clock}(i)=[\mathrm{UTCr}-\mathrm{UTC}(k)]+[\mathrm{UTC}(k)-\operatorname{Clock}(i)](1)$

Fit clock frequency by the least square linearfitting method according to $\mathrm{UTCr}-\operatorname{Clock}(i)$.The length of an interval of frequency fitting is 28 days. Calculate the atomic time scale during the interval of 7 days that from this published time of UTCr to the next released time.

\section{ALGORITHM OF ATOMIC TIME SCALE}

In this paper, Algos algorithm is considered as the algorithm of the atomic time scale calculation[5].

Assume that a laboratory has $\mathrm{N}$ atomic clocks: at time $\boldsymbol{t}$, for the clock $\mathrm{H}_{\mathrm{j}}$, the measurement is $\mathrm{x}_{\mathrm{ij}}(t)$.

$\mathrm{x}_{\mathrm{ij}}(t)=\mathrm{h}_{\mathrm{j}}(t)-\mathrm{h}_{\mathrm{i}}(t)$

The definition of TA is

$\mathrm{TA}(t)=\sum_{\mathrm{i}=1}^{\mathrm{N}} \mathrm{w}_{\mathrm{i}}(t)\left[\mathrm{h}_{\mathrm{i}}(t)-\mathrm{h}_{\mathrm{i}}{ }^{\prime}(t)\right]$

Where, $\mathrm{w}_{\mathrm{i}}(t)$ is the weight assigned to clock $\mathrm{H}_{\mathrm{i}} ; \mathrm{h}_{\mathrm{i}}{ }^{\prime}(t)$ is a correction term of reading of clock $\mathrm{H}_{\mathrm{i}}$ at time $t$, in order to ensure the continuity of time and frequency when the weight assigned to each clock or the number of clocks participating in the calculation are changed.
In fact, because there is no ideal time, $\mathrm{h}_{\mathrm{i}}(t)$ can not be obtained directly. Assume that the time difference between the reading of clock $\mathrm{H}_{\mathrm{i}}$ and $\mathrm{TA}(t)$ is [6]

$\mathrm{x}_{\mathrm{i}}(t)=\mathrm{TA}(t)-\mathrm{h}_{\mathrm{i}}(t), \quad \mathrm{i}=1, \ldots, \mathrm{N}$

From (2), (3) and (4), we can obtain

$\left\{\begin{array}{l}\sum_{\mathrm{i}=1}^{\mathrm{N}} \mathrm{w}_{\mathrm{i}}(t) \mathrm{x}_{\mathrm{i}}(t)=\sum_{\mathrm{i}=1}^{\mathrm{N}} \mathrm{w}_{\mathrm{i}}(t) \mathrm{h}_{\mathrm{i}}{ }^{\prime}(t) \\ \mathrm{x}_{\mathrm{ij}}(t)=\mathrm{h}_{\mathrm{j}}(t)-\mathrm{h}_{\mathrm{i}}(t), \mathrm{i} \neq \mathrm{j}\end{array}\right.$

Equation (5) has $\mathrm{N}$ equations and $\mathrm{N}$ unknown numbers, so it can be solved.

$t=t_{0}+\mathrm{nT} / 6, \mathrm{n}=0,1, \ldots, 6$

Where, the value of $\mathrm{T}$ is 7 days; $t_{0}$ is the last time of the last calculation interval. Also, $t_{0}$ is the first time of the current calculation interval.

$\mathrm{h}_{\mathrm{i}}{ }^{\prime}(t)$ is obtained from the linear prediction, the expression is

$\mathrm{h}_{\mathrm{i}}{ }^{\prime}(t)=\mathrm{x}_{\mathrm{i}}\left(t_{0}\right)+\mathrm{y}_{\mathrm{i}}{ }^{\prime}(t)\left(t-t_{0}\right)$

Where, $\mathrm{x}_{\mathrm{i}}\left(t_{0}\right)=\mathrm{TA}\left(t_{0}\right)-\mathrm{h}_{\mathrm{i}}\left(t_{0}\right)$ is known; $\mathrm{y}_{\mathrm{i}}{ }^{\prime}(t)$ is a frequency prediction value of clock $\mathrm{H}_{\mathrm{i}}$.

In actual calculation of $\operatorname{UTC}(k)-\mathrm{TA}^{\prime}(k)$, calculation cycle is 7 days and the length of calculation is 7 days, too. The length of frequency fitting interval is 28 days. $\operatorname{UTC}(k)-\mathrm{TA}^{\prime}(k)$ is updated once a day and clock frequency is updated every 7 day

$\operatorname{UTC}(k)-\operatorname{TA}^{\prime}(k)=\sum_{\mathrm{i}=1}^{\mathrm{N}} \mathrm{w}_{\mathrm{i}}(t)\left[(\operatorname{UTC}(k)-\operatorname{Clock}(i))-\mathrm{y}_{\mathrm{i}}{ }^{\prime}(t)\left(t-t_{0}\right)\right]$

Where, $\operatorname{UTC}(k)-\operatorname{Clock}(i)$ is the data of atomic clocks at 0 o'clock every day.

The weight of clock $\mathrm{H}_{\mathrm{i}}$ is kept invariant during the calculation interval $\left[t_{0}, t_{0}+\mathrm{T}\right]$.The weight is calculated as follows.

$\mathrm{w}_{\mathrm{i}}(t)=\frac{\frac{1}{\sigma_{\mathrm{i}}^{2}}}{\sum_{\mathrm{k}=1}^{\mathrm{N}} \frac{1}{\sigma_{\mathrm{k}}^{2}}}, \mathrm{k}=1, \ldots, \mathrm{N}$

Where, $\sigma_{\mathrm{i}}^{2}$ is the frequency stability.

If $\mathrm{w}_{\mathrm{i}}(t) \geq \mathrm{w}_{\text {max }}$, then [7]

$\mathrm{w}_{\mathrm{i}}(\mathrm{t})=\mathrm{w}_{\max }$

Where, $\mathrm{w}_{\max }=\mathrm{A} / \mathrm{N}$ is the maximum weight; $\mathrm{N}$ is the number of clocks participating in the calculation, A is a empirical constant, taking 1.5 in this paper.

$\sum_{\mathrm{i}=1}^{\mathrm{N}} \mathrm{w}_{\mathrm{i}}(t)=1$ 


\section{THE RESULTS}

The new method and the traditional method are applied respectively to the calculation of the atomic time scale in this paper. The time scale is calculated with the data of NICT, NTSC, NIM which participate in the calculation of International Atomic Time (TAI). The results ( $\left.\operatorname{UTC}(k)-\mathrm{TA}^{\prime}(k)\right)$ of two methods are compared with UTC $(k)-\mathrm{UTC}$, respectively. Thus, compare the superiorityinferiority of the new method and the traditional method in this paper.

\subsection{NICT}

Compare the time difference between UTC-UTC(NICT) and UTCr - UTC(NICT) (56628 56748).

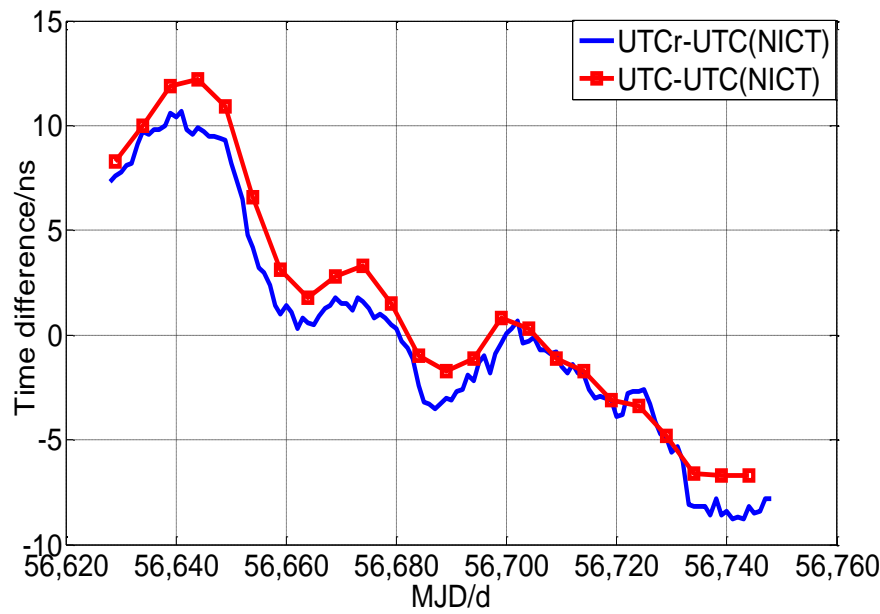

Figure 2.Comparison of the time difference between UTC-UTC(NICT) and UTCr-UTC(NICT)

Calculate the time difference of UTC(NICT) - TA'(NICT) and compare the time difference with UTC(NICT)-UTC by the new method and the traditional method, respectively. (MJD: 56659 56730)

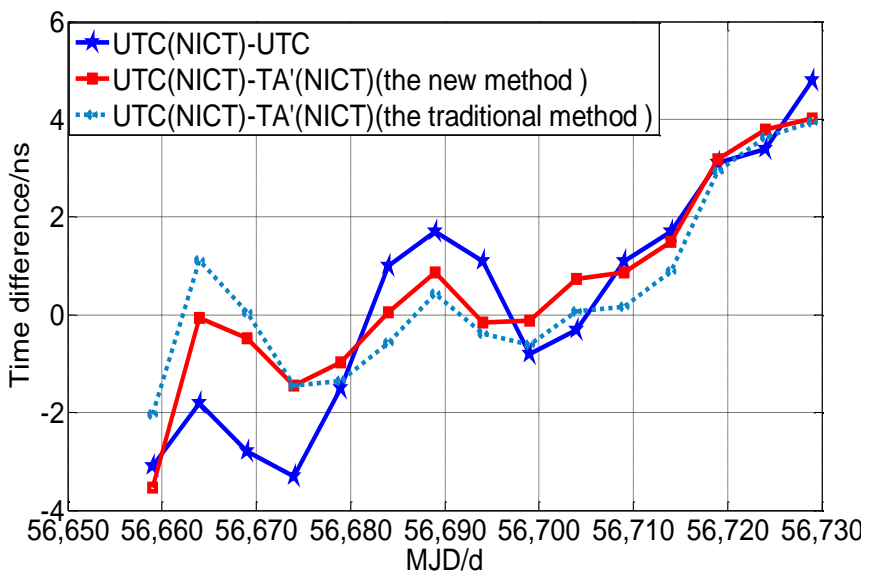

Figure 3. Comparison of the time difference between UTC(NICT) - TA'(NICT) and UTC(NICT) - UTC
Table 1.The deviation value between UTC(NICT)-TA'(NICT) and UTC-UTC(NICT) of different methods

\begin{tabular}{|l|c|c|}
\hline & The new method & The traditional method \\
\hline RMS(ns) & 1.2442 & 1.6106 \\
\hline
\end{tabular}

\subsection{NTSC}

Compare of the time difference between UTC-UTC(NTSC) and UTCr - UTC(NTSC) (56628 567 48).

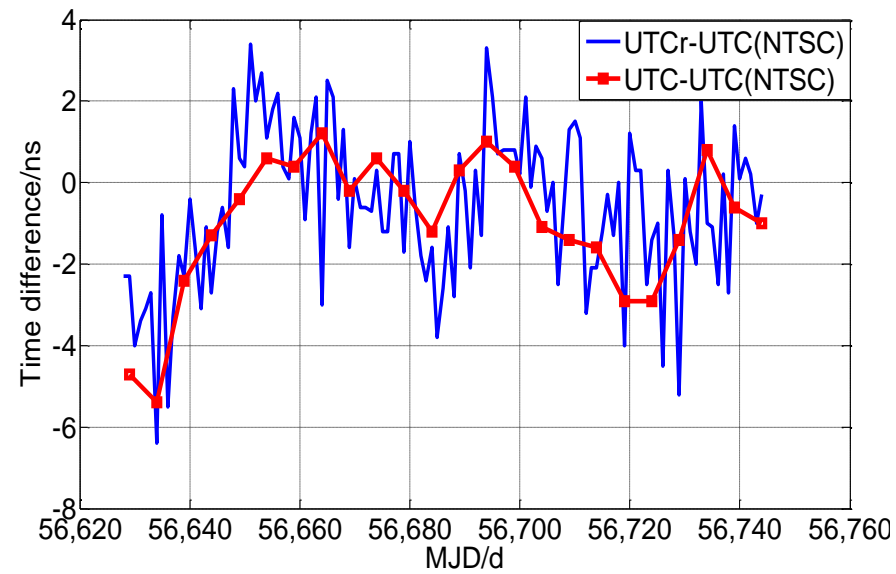

Figure 4. Comparison of the time difference between UTC-UTC(NTSC) and UTCr-UTC(NTSC)

Calculate the time difference of UTC(NTSC) - TA'(NTSC) and compare the time difference with UTC(NTSC)-UTC by the new method and the traditional method, respectively. (MJD: 56659 56730)

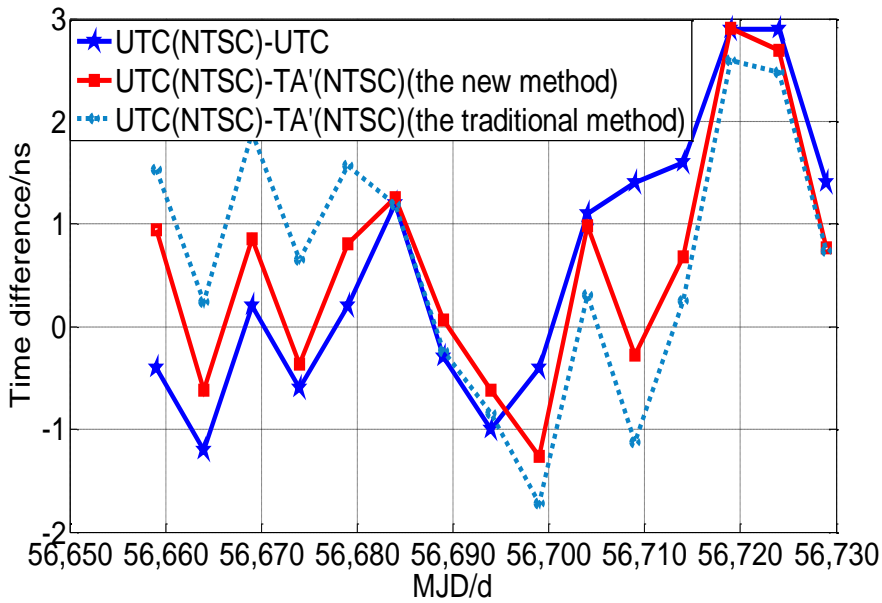

Figure 5. Comparison of the time difference between UTC(NTSC) - TA'(NTSC) and UTC - UTC(NTSC)

Table 2. The deviation value between UTC(NTSC)-TA'(NTSC) and UTC-UTC(NTSC) of different methods

\begin{tabular}{|c|c|c|}
\hline & The new method & The traditional method \\
\hline RMS(ns) & 0.7897 & 1.3788 \\
\hline
\end{tabular}




\subsection{NIM}

Compare of the time difference between UTC - UTC(NIM) and UTCr - UTC(NIM) (56628 56748).

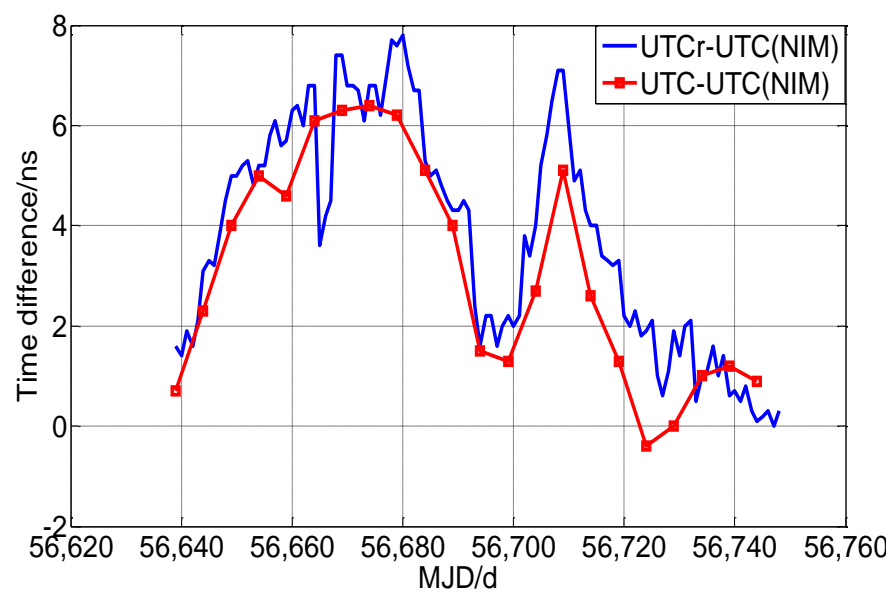

Figure 6. Comparison of the time difference between UTC-UTC(NIM) and UTCr-UTC(NIM)

Calculate the time difference of UTC(NIM)-TA'(NIM) and compare the time difference with UTC(NIM)-UTC by the new method and the traditional method, respectively. (MJD: 56659 56730)

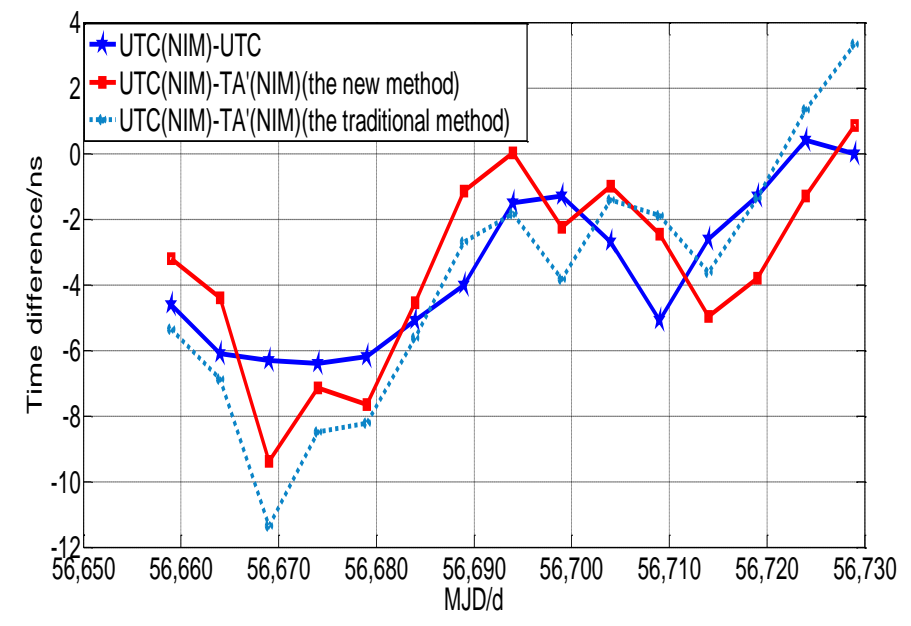

Figure 7. Comparison of the time difference between UTC(NIM) - TA'(NIM) and UTC-UTC(NIM)
Table 3. The deviation value between UTC(NIM)-TA'(NIM) and UTC-UTC(NIM) of different methods

\begin{tabular}{|l|c|c|}
\hline & The new method & The traditional method \\
\hline RMS(ns) & 1.8086 & 2.1306 \\
\hline
\end{tabular}

\section{CONCLUSION}

A dynamic prediction method of atomic clock frequency based on UTCr is proposed in this paper. The new frequency prediction method and the traditional frequency prediction method are applied respectively to the calculation of the atomic time scale by using the data of three laboratories (NICT, NTSC, NIM). The results ( UTC(k)-TA'(k)) of two methods are compared with UTC(k)-UTC by drawing and calculating the RMS of the difference curve, respectively. The result shows that the new method is more available than the traditional method. It improves greatly the quasi-real time of frequency prediction, provides more reliable reference for frequency steering and contributes to getting better stability and accuracy of UTC(k) .

\section{REFERENCES}

[1] www.bipm.org/jsp/en/TimeFtp.jsp?TypePub=publication

[2] G Petit, F Arias, A Harmegnies, G Panfilo and L Tisserand, UTCr: a rapid realization of UTC, Metrologia51 (2014) 33-39.

[3] Arias F., Harmegnies A., Jiang Z., Konaté H., Lewandowski W., Panfilo G., Petit G., Tisserand L., UTCr: a rapid realization of UTC, Proc. EFTF 2012, 2012, 24-27.

[4] Yuan Haibo. Dynamic grey-autoregressive model of an atomic clock. Metrologia 45(2008)S1-S5.

[5] Qi Guanrong. Time science foundation. Beijing: University of Chinese Academy of Sciences.

[6] He Ruizhen. The method of automatic analysis of punctual information and software design. Beijing: Higher Education Press, 2011.

[7] Gianna Panfilo, The new prediction algorithm for UTCr: application and results. FFTF (2012)242-246. 